\title{
Producción científica en innovación: Revisión sistemática de literatura
}

\begin{abstract}
Ariel Alonso de la Torre Ramos ${ }^{a}$, David Cortés Sáenz ${ }^{b}$, Fausto Enrique Aguirre Escárcega $^{c}$, Alibell Carolina Matheus Marín ${ }^{d}$

${ }^{a}$ Doctorante en Diseño, Fabricación y Gestión de Proyectos Industriales en la Universidad Politécnica de Valencia (UPV) 2018-2021. (Profesor titular en la Universidad Autónoma de Ciudad Juárez, Juárez, México, ariel.delatorre@uacj.mx), ${ }^{b}$ Doctorado en Proyectos de Innovación Tecnológica (Profesor titular en la Universidad Autónoma de Ciudad Juárez , Juárez, México, david.cortes@uacj.mx), ${ }^{c}$ Doctorado en Filosofía del Interior Arquitectónico (Profesor titular en la Universidad Autónoma de Ciudad Juárez, Juárez, México, fausto.aguirre@uacj.mx), ${ }^{\mathrm{d}}$ Maestranda en Diseño y Desarrollo de Producto (Universidad de Ciudad Juárez , Juárez, México, al183335@alumnos.uacj.mx).
\end{abstract}

\section{Resumen}

El presente estudio tiene como objetivo analizar artículos científicos de acceso abierto (Open Access), con el fin de identificarla la producción cientifica que aborden temas de innovación. El método para llevar acabo el estudio fue una revisión sistemática de literatura (SLR) el cual consta de 3 fases. La fase 1 planificación; donde se orienta a seleccionar bases de datos pertinentes y la búsqueda a partir de palabras claves. La fase 2 conducción; se evalúa y extraen los datos con base a los objetivos planteados. Fase 3 informe de resultados; se enfoca a validar los criterios considerando calidad y pertinencia de los estudios encontrados.

Hacer una búsqueda de la literatura para obtener cómo se define la innovación puede ser complicado, ya que la palabra innovación se emplea en diversos contextos, sectores y disciplinas, por lo cual dependiendo el enfoque de tema al que se pretenda indagar se obtendrán distintas narraciones sobre la definición de la innovación.

Como resultado se obtuvo el análisis de 103 artículos publicados en acceso abierto, de enero 2017 a noviembre 2019, en las bases de datos de Web Of Science y Scopus, teniendo como hallazgo que las entidades de mayor publicación sobre el tema son la Unión Europea y los Estados Unidos de América, en lo que concierne a un enfoque de los sectores empresariales y académicos seguidos por el enfoque social y cultural. 
Palabras clave: Innovación; Tipo de Innovación; Concepto Innovación; Revisión Literatura

\section{Introducción}

En la ultima década el vocablo "innovación" se ha convertido en un referente adaptado a diferentes contextos, sectores y disciplinas, por lo que la definición de innovación tiende a variar según su objetivo o enfoque, la utilización de la palabra innovación se ve mayormente vinculada en los sectores empresariales, principalmente en los procesos involucrados en el desarrollo de productos, como son la fabricación, la comercialización y la organización en la empresa.

Si bien el tema de innovación puede resultar complejo, es necesario comprender de donde nace la palabra, así como entender su significado. Para ello se puede abordar el concepto de la RAE (2019), el cual menciona que "la palabra innovación proviene del latín innovatio cuyo significado es la creación o modificación de un producto, y su introducción en un mercado". Por lo tanto, la innovación puede estar presente en un producto con cualquiera de estas características: creativo, novedoso y original. Ahora bien, para considerar algo innovador, a pesar de contar con las características mencionadas, este tiene que causar un efecto positivo en alguno de estos bienes: económico, tecnológico, organización o procesos.

Distintos autores han presentado definiciones sobre el tema, tenemos por ejemplo a Schumpeter (1934) el cual define la innovación abarcando cinco casos: la introducción en el mercado de un nuevo bien, un nuevo método de producción, la apertura de un nuevo mercado en un país, la conquista de una nueva fuente de suministro de materias primas o de productos y por último la implantación de una nueva estructura en un mercado.

Otros autores pueden definir a la innovación como la transformación de conocimiento en nuevos productos y servicios, donde Nelson, (1997) sustenta que "no es un evento aislado, sino la respuesta continua a circunstancias cambiantes". Asimismo, una de las definiciones de carácter general de Corma, (2013) es "la innovación como la trasformación de ideas y conocimientos en productos, procesos o servicios que son renovados para el mercado, para con ello satisfacer las necesidades de los ciudadanos, empresas y organizaciones públicas".

El referente más importante en la temática de innovación es el Manual de Oslo, propuesto por la Organización para la Cooperación y el Desarrollo Económico (2005), el cual es una guía para la realización de mediciones y estudios de actividades científicas y tecnológicas en las empresas, asimismo define conceptos y clarifica las actividades consideradas como innovadoras. Dicho manual menciona que "se entiende por innovación la concepción e 
implantación de cambios significativos en el producto, el proceso, el marketing o la organización de la empresa con el propósito de mejorar los resultados”.

Ahora bien, la innovación puede estar vinculada a cualquier área de la economía, incluyendo los servicios públicos tales como sector salud o el sector de la educación. Sin embargo, las principales definiciones y enfoques de estudio de innovación se observan en el sector empresarial. Por otra parte, es importante destacar el papel de las universidades dentro de la innovación, el cual se considera como un vinculo de adquisición y transferencia de conocimiento y tecnología , así como una fuente esencial del proceso de innovación debido a su libre acceso a la información (M.Oslo, 2005).

Tomando en cuanta lo anterior, se puede considerar que hay trabajo por realizar en materia de innovación dentro del sector de la educación, desde luego es necesario identificar en primera instancia que información se tiene en existencia dentro del marco científico para así conocer el universo de publicaciones dentro del sector.

\section{Metodología}

El objetivo general de la investigación es identificar el universo de las producciones científicas que aborden temas de innovación atreves el análisis de diferentes artículos que se encuentran en acceso abierto. Asimismo, obtener información sobre la distribución geográfica de las publicaciones, el enfoque de las publicaciones y la incidencia de tipos de innovación.

El método para llevar acabo el estudio fue una revisión sistemática de literatura (SLR) con el cual se realizó la exploración de los documentos en las bases de datos de Web Of Science y Scopus, dicho método fue basado en estudios previos de Soledad Ramírez \& García Peñalvo (2018) en el cual abordan una metodología para el análisis bibliométrico por medio la revisión de literatura sistemática.

El estudio consta de 3 fases, las cuales se estructuran de la siguiente forma; La fase 1 planificación; donde se orienta a seleccionar bases de datos pertinentes y la búsqueda a partir de palabras claves (Barbara, 2004). La fase 2 conducción; se evalúa y extraen los datos con base a los objetivos planteados (Bossuyt et al., 2013). Fase 3 informe de resultados; se enfoca a validar los criterios considerando calidad y pertinencia de los estudios encontrados (Booth, Wright, \& Outhwaite, 2010). 


\subsection{Objeto de estudio}

Como objeto de estudio, se tiene la innovación. El fin es identificar información y publicaciones realizadas en torno a innovación. Para la revisión, selección y evaluación de los estudios pertinentes se desarrollo el siguiente protocolo:

- Acervos de búsqueda : Base de datos Web Of Science y base de datos Scopus.

- Palabras clave y criterios de búsqueda: Concepto de innovación (Innovation Concept), Definición de innovación (Innovation Definition), Tipo de innovación (Innovation Type).

- Criterios de inclusión y exclusión:

- Temporalidad del estudio: Periodo enero 2017 a noviembre del 2019.

- Categoría de documento: Documentos de acceso abierto (open acces) como : artículos científicos; artículos indexados; artículos de revista.

- Campo de estudio : Innovación.

○ Idioma de búsqueda: inglés.

\subsection{Limitaciones del estudio}

Para la realización de la revisión sistemática de literatura, se hace exclusivamente a los resultados obtenidos a través de las bases de datos de Web Of Science (BD-W) y Scopus (BD-S). Cabe destacar que se realizaron tres búsquedas en las cuales se fueron depurando publicaciones arrojadas por las BD-W y BD-S, la primera búsqueda se basó en las palabras claves, en la segunda búsqueda se descartaron aquellas publicaciones que no son de acceso abierto y publicaciones que duplican en ambas bases de datos, en la tercera búsqueda se descartaron las publicaciones con poco aporte al objeto de estudio por el área temática como temas de minería y ciencias médicas.

\section{Resultados}

Aclarados los puntos anteriores, se presentan los resultados obtenidos en la revisión sistemática de literatura. En la Tabla 1, se puede observar los resultados de las tres búsquedas delimitadas en las bases de datos BD-W y BD-S, donde se muestra el universo de documentos localizados, teniendo los documentos que se incluyeron para la exploración y los que fueron descartados de acuerdo con los criterios antes mencionados. Como resultado se obtuvo el análisis de 103 artículos publicados en acceso abierto, de enero 2017 a noviembre 2019, de los cuales se descartaron 56, y obteniendo un total de 47 publicaciones para analizar. 
Tabla 1. Principales incidencias de innovación en los buscadores de BD-S y BD-S

\begin{tabular}{|c|c|c|c|c|c|c|c|c|c|}
\hline \multicolumn{10}{|c|}{ Principales incidencias de innovación en los buscadores de BD-S y BD-S } \\
\hline \multicolumn{5}{|c|}{ Primera búsqueda } & \multicolumn{3}{|c|}{ Segunda Búsqueda } & \multicolumn{2}{|c|}{ Tercera búsqueda } \\
\hline \multirow[t]{2}{*}{$\begin{array}{l}\text { Palabras } \\
\text { Clave }\end{array}$} & \multicolumn{2}{|c|}{$\begin{array}{l}\text { Artículos con } \\
\text { "Innovation } \\
\text { Concept"o } \\
\text { "Innovation } \\
\text { Definition" o } \\
\text { "Innovation type" }\end{array}$} & \multicolumn{2}{|c|}{$\begin{array}{c}\text { Articulos delimitados } \\
\text { en revistas de Acceso } \\
\text { Directo }\end{array}$} & \multirow[t]{2}{*}{$\begin{array}{l}\text { Sub- } \\
\text { total de } \\
\text { las } 2 \\
\text { base de } \\
\text { datos } \\
\text { en } \\
\text { abierto }\end{array}$} & \multirow[t]{2}{*}{$\begin{array}{l}\text { Duplicad } \\
\text { os }\end{array}$} & \multirow[t]{2}{*}{$\begin{array}{l}\text { Open } \\
\text { Acces } \\
\text { en } \\
\text { BDW- } \\
\text { BDS }\end{array}$} & \multirow[t]{2}{*}{$\begin{array}{l}\text { Eliminados } \\
\text { por no } \\
\text { abordar } \\
\text { temas de } \\
\text { innovación }\end{array}$} & \multirow[t]{2}{*}{$\begin{array}{c}\text { Seleccionados } \\
\text { por } \\
\text { corresponder } \\
\text { temas de } \\
\text { innovación. }\end{array}$} \\
\hline & $\begin{array}{c}\text { Base de } \\
\text { Datos } \\
\text { SCOPuS }\end{array}$ & $\begin{array}{c}\text { Base de } \\
\text { Datos } \\
\text { wos }\end{array}$ & $\begin{array}{c}\text { Base de } \\
\text { Datos } \\
\text { Scopus }\end{array}$ & $\begin{array}{c}\text { Base de } \\
\text { Datos } \\
\text { wos }\end{array}$ & & & & & \\
\hline $\begin{array}{c}\text { Innovation } \\
\text { Concept }\end{array}$ & 127 & 149 & 32 & 18 & 50 & 2 & 48 & 32 & 16 \\
\hline $\begin{array}{l}\text { Innovation } \\
\text { Definition }\end{array}$ & 18 & 42 & 6 & 7 & 13 & 2 & 11 & 2 & 9 \\
\hline $\begin{array}{c}\text { Innovation } \\
\text { Type }\end{array}$ & 109 & 161 & 20 & 26 & 46 & 2 & 44 & 22 & 22 \\
\hline Total: & & & & & & & 103 & & 47 \\
\hline
\end{tabular}

Fuente: Elaboración propia a partir de datos analizados

De los 47 artículos se encontró que los países con publicaciones son: España (11), Estados Unidos (8), Suecia (6), Países Bajos (5) Reino Unido (4), China (4), India (1), Ucrania (1), Taiwán (1), Malasia (1), Finlandia (1), Turquía (1), Brasil(1), Rusia (1) y Australia (1). En la Figura 1 se representa la distribución geográfica, donde los círculos exponen gráficamente la cantidad de publicaciones que se han encontrado en el estudio.

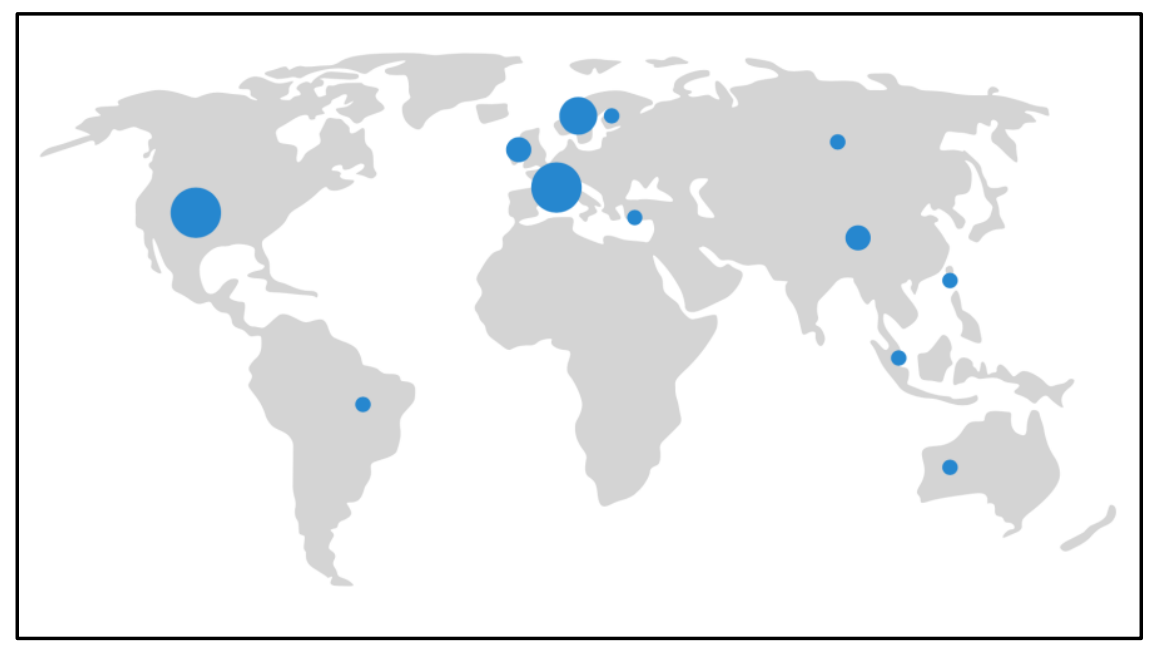

Figura 1. Publicaciones por país.

Fuente: Elaboración propia a partir de datos analizados. 
Se realizó un análisis de contenido para identificar el enfoque de las publicaciones. En la Figura 2 se observa un diagrama que indica el número de artículos y año de publicación de acuerdo con los siguientes enfoques : social, académico, cultural y empresarial.

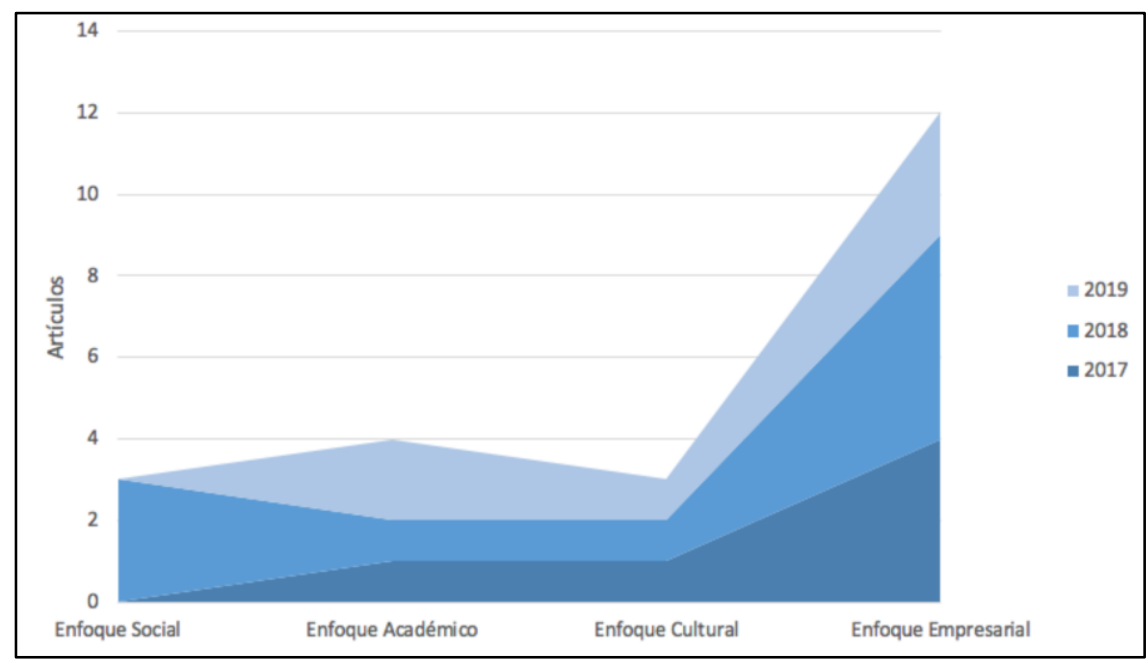

Figura 2. Publicaciones por enfoques

Fuente: Elaboración propia a partir de datos analizados.

Con los resultados anteriores se identificaron los tipos de innovación de mayor incidencia en base a conceptos y definiciones identificados en los documentos analizados, estos datos se pueden observar en la Figura 3 donde a mayor tamaño de la letra expone gráficamente la incidencia del tipo de innovación identificado.

\section{Proceso de Innovación Modele de negocoidinnovación} Innovación sostenible Chinnovation Sistema de innovación Innovación modular Innovación sistemática Intermediarios de innovación Innovación Abierta In Inovación de producto
Innovación Rendimiento de la innovación Innovación colaborativa Innovación Social Innovación tecnológica Innovación orientada a la sostenibilidad, Innovación Cultural Innovación de marketing Diseño e innovación In

\section{Innovación sostenible Innovación arquitectónica Innovación conceptual Innovación Organizacional}

Figura 3. Tipos de innovación identificados

Fuente: Elaboración propia a partir de datos analizados. 


\section{Conclusiones}

El estudio declara que la revisión sistemática de literatura fue realizada en las bases de datos de Web Of Science y Scopus, con la temporalidad de enero de 2017 a noviembre del 2019, con la objetivo de identificar como se define la innovación a partir de indicadores que determinen el enfoque y tipo de innovación; de esta forma se pretende ampliar el entendimiento en el campo de estudio de la innovación.

Dentro del estudio se destacan los siguientes puntos los cuales son el resultado del proceso metodológico antes mencionado:

- En los tres años que abarca el estudio, se encontraron un total de 606 documentos dentro de las bases de datos de Web Of Science y Scopus, los cuales de la primera depuración resultaron 109 documentos de acceso abierto que abordaban el objeto de estudio.

- En la segunda depuración donde se eliminaron los documentos duplicados en ambas bases de datos dio como resultado un total de 103 documentos para su análisis a detalle.

- En la tercera depuración se descartaron los documentos que no apartaban relevancia al objeto de estudio.

- Los principales enfoques que abordan el enfoque de estudio son social, académico, cultural y empresarial. Donde se puede observar que en año 2017 una mayor incidencia en el enfoque empresarial y nula en el enfoque social, en el año 2018 se observa un incremento en el enfoque social, cultural y académico, manteniendo la mayor incidencia en el enfoque empresarial, de la misma forma se observa un comportamiento similar en el año 2019, con incremento en el enfoque académico.

- Los principales tipos de innovación identificados fueron : Innovación de producto, innovación radical, innovación en procesos e innovación sostenible.

\section{Referencias}

Barbara, K. (Keele U. (2004). Procedures for Performing Systematic Reviews. In Keele University Technical Report TR/SE-0401. Retrieved from http://www.inf.ufsc.br/ aldo.vw/kitchenham.pdf

Booth, A. M., Wright, K. E., \& Outhwaite, H. (2010). Centre for Reviews and Dissemination databases: Value, content, and developments. International Journal of Technology Assessment in Health Care, 26(4), 470-472. https://doi.org/10.1017/S0266462310000978

Bossuyt, P., Davenport, C., Deeks, J., Hyde, C., Leeflang, M., \& Scholten, R. (2013). Cochrane Handbook for Systematic Reviews of Diagnostic Test Accuracy Chapter 11 Interpreting results 
and drawing conclusions. 1-31. Retrieved from http://srdta.cochrane.org/.

Corma, F. (2013). Innovación, innovadores y empresa innovadora. Madrid: Ediciones Díaz de Santos, S.A.

Nelson, B. (1997) 1001 formas de motivar a los empleados. Editorial Norma. Ecuador.

Organización para la Cooperación y el Desarrollo Económicos OECD (2005)

Manual de Oslo. Guía para la recogida e interpretación de datos sobre innovación. España: Grupo Tragsa.

Schumpeter, Joseph A. The Theory of Economic Development. McGraw-Hill, Nueva York. 1934.

Soledad Ramírez, M. (Escuela de H. y E. del T. de M. M., \& García Peñalvo, F. J. (Departamento de I. y A. en la U. de S. E. (2018). Co-creación e innovación abierta: Revisión sistemática de literatura. Revista Cientifica de Educomunicación, 54, 9-18. Retrieved from https://doi.org/10.3916/C54-2018-01

Real Academia Española, RAE (2019) Innovación | Diccionario de la lengua española. Recuperado 12 de junio de 2020 de: https://dle.rae.es/innovaci\%C3\%B3n 